(2) Open Access Full Text Article

\title{
Metastatic medullary thyroid cancer: a dramatic response to a systemic chemotherapy (temozolomide and capecitabine) regimen
}

This article was published in the following Dove Press journal:

OncoTargets and Therapy

13 May 2015

Number of times this article has been viewed

\author{
Sahin Lacin \\ Ece Esin \\ Yusuf Karakas \\ Suayib Yalcin \\ Department of Medical Oncology, \\ Institute of Cancer, Hacettepe \\ University, Ankara, Turkey
}

Correspondence: Suayib Yalcin Department of Medical Oncology, Institute of Cancer, Hacettepe University, Ankara, Turkey

Tel +90 3/2 3092904

Email suayibyalcin@gmail.com

\begin{abstract}
A 40-year-old male patient presented with increasing serum levels of calcitonin and CEA. He underwent potential curative surgery for medullary thyroid carcinoma, 3 years ago and then 7 months later he had metastasectomy and cervical lymph node dissection for recurrent disease. On admission he had multiple metastatic skin nodules on the chest wall and positron emission tomography-computed tomography revealed multiple visceral metastases as well. The patient had not received any systemic treatment up to that time; therefore, we considered systemic treatment with the new tyrosine kinase inhibitors (vandetanib, cabozantinib, etc). However, since these drugs are only available after cytotoxic chemotherapy, we started temozolomide and capecitabine chemotherapy. After two courses of the treatment all skin nodules disappeared and CEA and calcitonin levels normalized, radiological imaging showed a good partial response.
\end{abstract}

Keywords: medullary thyroid cancer, capecitabine, temozolomide, chemotherapy

\section{Introduction}

Thyroid cancer accounts for $1 \%-2 \%$ of all malignancies, representing the most common endocrine malignancy. ${ }^{1}$ Medullary thyroid carcinoma (MTC) accounts for $5 \%-10 \%$ of cases of all thyroid cancers. ${ }^{2}$ Histological subtypes of thyroid cancer have different embryological origins; MTC originates from parafollicular cells of the ultimobranchial body, derived from the fourth pharyngeal pouch. ${ }^{3}$ They occur both as sporadic tumors and as a component of multiple endocrine neoplasia type 2 . Hereditary MTC is a component of multiple endocrine neoplasia type 2 but also presents as familial MTC. Mutations of the proto-oncogene, RET, found on chromosome 10q11, are present in more than $95 \%$ of hereditary MTCs and approximately $25 \%$ of sporadic MTCs. ${ }^{4}$ They secrete calcitonin and carcinoembryogenic antigen (CEA), both of which can serve as tumor markers. The primary treatment for MTC is aggressive surgical resection. The role of external-beam radiotherapy is limited and radioiodine treatment or thyroid-stimulating hormone-suppression is not indicated because these tumors do not concentrate iodine.

Patients with progressive or symptomatic metastatic disease who cannot be treated with surgery or radiotherapy should be considered as candidates for systemic therapy. Treatment with either cytotoxic chemotherapy or biologic response modifiers may provide some benefit for occasional patients. New targeted therapies are emerging as effective interventions for progressive disease. Now chemotherapy is reserved for patients who have failed or are ineligible for targeted therapies. 


\section{Case}

A 40-year-old male patient presented to a hospital in December 2011 with a right-sided thyroid mass and hoarseness. An ultrasound scan (USS) and fine needle aspiration cytology was consistent with MTC. The blood laboratory analysis of the patient revealed high serum levels of calcitonin and CEA, $222 \mathrm{pg} / \mathrm{mL}$ and $18 \mathrm{ng} / \mathrm{mL}$, respectively. In December 2011, the patient underwent a total thyroidectomy and bilateral neck lymphatic dissection with a good recovery. Histological analysis of this specimen revealed a $17 \mathrm{~mm}$ MTC. In total 49 lymph nodes were removed from the right central, paratracheal, right lateral neck, and 13 lymph nodes contained malignant deposits. These were positive for MTC (12 lymph nodes from the right and one lymph node from the left cervical). Tumor tissue examination for the RET oncogene mutations was negative and no familial or personal history was positive for a genetic syndrome. The postoperative serum calcitonin and CEA levels were $1.99 \mathrm{pg} / \mathrm{mL}$ (normal range: $0-18 \mathrm{pg} / \mathrm{mL}$ ) and $0.9 \mathrm{ng} / \mathrm{mL}$ (normal range: $0-3 \mathrm{ng} / \mathrm{mL}$ non-smoker), respectively. A USS of the neck did not reveal lymphadenopathy. Following normal urinary catecholamine, metanephrine determination the patient proceeded to a surveillance program. The patient made a good recovery with a normal serum calcium on no supplements at 2 weeks follow-up. However, in the subsequent months the calcitonin levels began to rise to prereoperative surgery levels suggesting low grade persistent disease. A computed tomography (CT) scan and USS of the neck were performed in July 2012 revealing only cytologically proven small metastatic lymph nodes. The patient underwent a repeat uncomplicated selective neck lymph dissection. Histological analysis confirmed that lesions showed features consistent with metastatic MTC. Immunocytochemistry revealed calcitonin expression and nuclear TTF-1 expression supporting MTC. In the subsequent months serum calcitonin and CEA levels began to rise. Skin nodules began to grow and by August 2014, serum calcitonin and CEA levels reached $411 \mathrm{pg} / \mathrm{mL}$ and $31.51 \mathrm{ng} / \mathrm{mL}$. A fluoro-deoxy-D-glucosepositron emission tomography (FDG-PET) scan of the whole body, performed in August 2014, revealed widespread evidence of metabolically active disease involving the right retropharyngeal space, upper right side of larynx, right superficial, depth axillar conglomerated lymph nodes, paraesophageal lymph nodes, IVa and VIII liver segments, aortocaval, peripancreatic, para-aortic, and pericaval areas consistent with widespread disease. Since surgery was not possible we considered the use of tyrosine kinase inhibitors (TKIs) (vandetanib, cabozantinib, etc) as first line therapy but these drugs are only available after systemic cytotoxic treatment in Turkey with special permission. Therefore, we treated our patient with capecitabine $\left(1,700 \mathrm{mg} / \mathrm{m}^{2}\right)$ from day 1 to 14 and temozolomide $\left(200 \mathrm{mg} / \mathrm{m}^{2}\right)$ from day 10 to 14 every 4 weeks. Assessments of tumor measurements, serum calcitonin, and CEA were made before treatment and followed-up. After two courses of treatment, the patient was referred to the clinic in December 2014 with improved performance and was asymptomatic with normal hemoglobin, calcium, urea, and creatinine levels. The calcitonin and CEA levels decreased to normal range (Figures 1 and 2). The radiologic imagines (thorax and abdominal CT) revealed significant shrinkage of axillary, para-aortic, and paracaval lymph nodes and shrinkage rate including liver lesions was more than $50 \%$. The chemotherapy was well tolerated, with no diarrhea or hematologic toxicities.

\section{Discussion}

In patients with progressive metastatic MTC, treatment with traditional cytotoxic agents provides limited benefit. Partial responses are reported in approximately 10\%-20\% of patients, but long-term responses are uncommon and cure is impossible. As in other tumors, tyrosine kinases play a significant role in the regulation of tumor proliferation, angiogenesis, invasion, and metastasis in MTC. Small molecule inhibitors of select tyrosine kinases have been of interest for the treatment of advanced MTC, given the oncogenic role of inherited and somatic mutations in the tyrosine kinase RET, as well as the contributory roles of tyrosine kinases in growth factor receptors such as VEGFR. ${ }^{5}$ These drugs partially

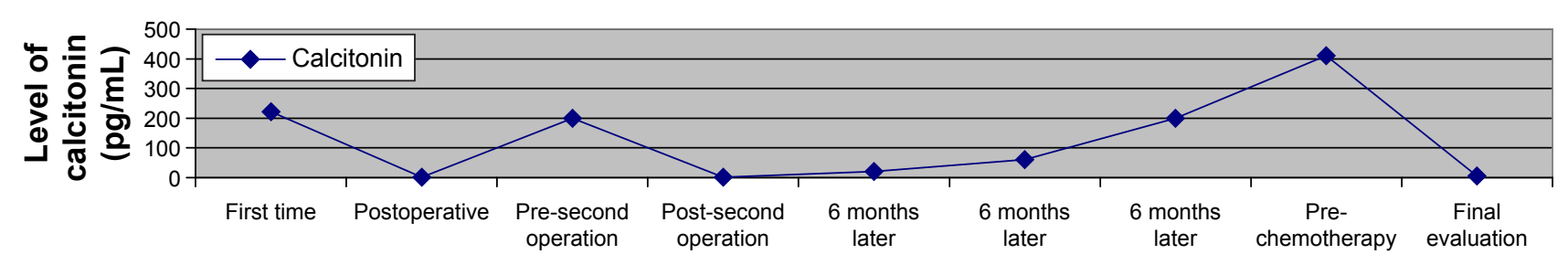

Follow-up (month)

Figure I Graphic shows level of calcitonin during follow-up of the patient. 


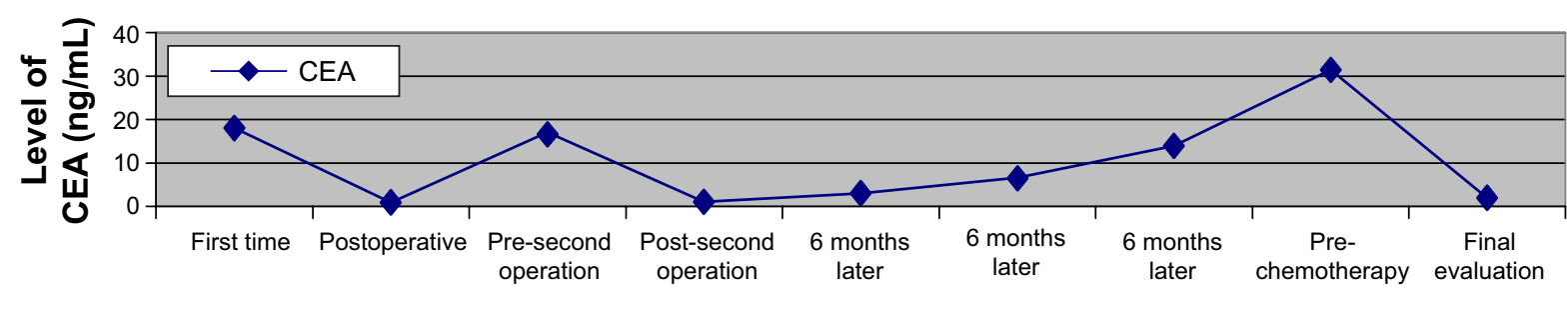

Follow-up (month)

Figure 2 Graphic shows level of CEA during follow-up of the patient.

inhibit multiple kinases at nanomolar concentrations and often affect multiple signaling pathways. So availability of effective TKIs is changing the standard approach in MTC, further limiting the role of cytotoxic agents. ${ }^{6}$ Thus, there is a trend toward using traditional cytotoxic agents as a salvage therapy in MTC or reserved for patients who are unable to participate in clinical trials or have failed or cannot tolerate kinase inhibitors. Most regimens for patients with MTC combine dacarbazine with other agents, including vincristine, 5-fluorouracil, cyclophosphamide, streptozocin, or doxorubicin, without significant advantage of one combination compared to another. The combination of cyclophosphamide $\left(750 \mathrm{mg} / \mathrm{m}^{2}\right)$, vincristine $\left(1.4 \mathrm{mg} / \mathrm{m}^{2}\right)$, and dacarbazine $\left(600 \mathrm{mg} / \mathrm{m}^{2}\right.$ daily for 2 days in each cycle) every 3 weeks was administered to seven patients with metastatic MTC. ${ }^{7}$ Two patients experienced $>50 \%$ shrinkage in tumor dimensions lasting more than 1 year, and two others had stable disease. Another regimen (repeating cycles of doxorubicin $60 \mathrm{mg} / \mathrm{m}^{2}$ on day 1 , and streptozocin $500 \mathrm{mg} / \mathrm{m}^{2}$ daily for 5 consecutive days, followed 4 weeks later, by fluorouracil $400 \mathrm{mg} / \mathrm{m}^{2}$, and dacarbazine, $200 \mathrm{mg} / \mathrm{m}^{2}$, daily for 5 consecutive days) was given to 20 patients with progressing distant metastases. ${ }^{8}$ Three patients (15\%) had partial responses lasting more than 18 months, and ten (50\%) were stable for at least 8 months. Toxicities of dacarbazine include neutropenia, thrombocytopenia, nausea, vomiting, and hepatotoxicity. Doxorubicin $\left(60-75 \mathrm{mg} / \mathrm{m}^{2}\right.$ every 3 weeks, or $15 \mathrm{mg} / \mathrm{m}^{2}$ weekly) has been approved by the US Food and Drug Administration (FDA) for the treatment of all histological types of metastatic thyroid carcinoma including MTC, but fewer than $30 \%$ of patients have an objective response, without any complete responses, and the duration is generally short. ${ }^{9,10}$ Temozolomide is an oral alkylating agent with a wide range of antitumor activity and relatively little toxicity. Both temozolomide and dacarbazine are prodrugs of the active alkylating agent 5-(3-methyltriazen-1-yl) imidazole-4-carboximide. Although dacarbazine requires metabolic activation, temozolomide spontaneously converts to 5-(3-methyltriazen-1-yl)imidazole-4-carboximide under physiologic conditions. Cytotoxicity of temozolomide is mediated principally through methylation of DNA at the $O 6$ position of guanine, although other mechanisms of actions have also been proposed. Temozolomide has demonstrated $100 \%$ oral bioavailability, and is therefore a better alternative to dacarbazine.

Recent small studies have suggested activity of the combination of temozolomide and capecitabine in some gastrointestinal neuroendocrine tumors (NETs). In one retrospective study of 18 patients with metastatic, welldifferentiated NETs, there was one complete radiographic response and one partial response among four patients with gastrointestinal NET. Notably, one of the responses was in a patient with a duodenal NET. ${ }^{11}$ In a report of a Phase II trial of temozolomide plus capecitabine, four of 12 patients with metastatic NETs originating in various sites were reported as having partial responses. ${ }^{12}$ However, the primary sites of these responding tumors were not reported. Larger, prospective studies are clearly needed to better assess the potential activity of temozolomide and capecitabine in NETs and to assess whether activity may differ depending on primary tumor site.

MTC is a well-differentiated NET, therefore MTC patients have so far been treated with drugs with demonstrated activity against other well-differentiated NETs. So far, some Phase II clinical trials using capecitabine in the treatment of metastatic MTC have been published. ${ }^{13-15}$ Temozolomide and capecitabine regimen is well tolerated at the dose of temozolomide $\left(200 \mathrm{mg} / \mathrm{m}^{2}\right)$ and capecitabine $\left(1,700 \mathrm{mg} / \mathrm{m}^{2}\right)$ per day. Diarrhea and hand-foot syndrome, two of the major adverse effects of capecitabine, were not observed. Serious hematological side effects of temozolomide were not reported either.

As far as we know, this is the first report demonstrating the clear activity of temozolomide and capecitabine in MTC. These drugs may represent a reasonable therapeutic alternative for patients with metastatic disease. So this case is important because it shows clear activity of this combination in MTC. 


\section{Disclosure}

The authors have no conflicts of interest to disclose.

\section{References}

1. Vanderpump MP. The epidemiology of thyroid disease. Br Med Bull. 2011;99:39-51.

2. Hundahl SA, Cady B, Cunningham MP, et al. Initial results from a prospective cohort study of 5583 cases of thyroid carcinoma treated in the United States during 1996. Cancer. 2000;89(1):202-217.

3. Ball DW. Medullary thyroid cancer: therapeutic targets and molecular markers. Curr Opin Oncol. 2007;19(1):18-23.

4. Liu Z, Falola J, Zhu X, et al. Antiproliferative effects of Src inhibition on medullary thyroid cancer. J Clin Endocrinol Metab. 2004;89(7): 3503-3509.

5. Nikiforova MN, Nikiforov YE. Molecular genetics of thyroid cancer: implications for diagnosis, treatment and prognosis. Expert Rev Mol Diagn. 2008;8(1):83-95.

6. Zhang J, Yang PL, Gray NS. Targeting cancer with small molecule kinase inhibitors. Nat Rev Cancer. 2009;9(1):28-39.

7. Wu LT, Averbuch SD, Ball DW, et al. Treatment of advanced medullary thyroid carcinoma with a combination of cyclophosphamide, vincristine, and dacarbazine. Cancer. 1994;73(2):432-436.

8. Nocera M, Baudin E, Pellegriti G, et al. Treatment of advanced medullary thyroid cancer with an alternating combination of doxorubicinstreptozocin and 5 FU-dacarbazine. Groupe d'Etude des Tumeurs à Calcitonine (GETC). Br J Cancer. 2000;83(6):715-718.
9. Shimaoka K, Schoenfeld DA, DeWys WD, Creech RH, DeConti R. A randomized trial of doxorubicin versus doxorubicin plus cisplatin in patients with advanced thyroid carcinoma. Cancer. 1985;56(9):2155-2160.

10. Porter AT, Ostrowski MJ. Medullary carcinoma of the thyroid treated by low-dose adriamycin. Br J Clin Pract. 1990;44:517.

11. Fine RL, Gulati AP, Krantz BA, et al. Capecitabine and temozolomide (CAPTEM) for metastatic, well-differentiated neuroendocrine cancers: The Pancreas Center at Columbia University experience. Cancer Chemother Pharmacol. 2013;71(3):663-670.

12. Fine RL, Gulati AP, Tsushima D, et al. Prospective phase II study of capecitabine and temozolomide (CAPTEM) for progressive, moderately, and well-differentiated metastatic neuroendocrine tumors. J Clin Oncol 32. 2014; (Suppl 3; abstr 179).

13. Fine RL, Fogelman DR, Schreibman SM. Effective treatment of neuroendocrine tumors with temozolomide and capecitabine. J Clin Oncol. $2005 ; 23(16 \mathrm{~S}): 4216$.

14. Hoff PM, Hoff AO, Phan AT, Sherman SI, Yao J, White N, et al. Phase I/II trial of capecitabine (C), dacarbazine (D) and imatinib (I) (CDI) for patients (pts) metastatic medullary thyroid carcinomas (MTC). J Clin Oncol. 2006;24(18S):13048.

15. Droz J, Baudin E, Medvedev V, et al. Activity of irofulven (IROF) combined with capecitabine (CAPE) in patients (pts) with advanced thyroid carcinoma: phase II international multicenter study (preliminary results). J Clin Oncol. 2006;24(18S):15511.
OncoTargets and Therapy

\section{Publish your work in this journal}

OncoTargets and Therapy is an international, peer-reviewed, open access journal focusing on the pathological basis of all cancers, potential targets for therapy and treatment protocols employed to improve the management of cancer patients. The journal also focuses on the impact of management programs and new therapeutic agents and protocols on

\section{Dovepress}

patient perspectives such as quality of life, adherence and satisfaction. The manuscript management system is completely online and includes a very quick and fair peer-review system, which is all easy to use. Visit http://www.dovepress.com/testimonials.php to read real quotes from published authors. 